13

\title{
Astronomical Molecular Spectroscopy
}

Timothy W. Schmidt

\section{1}

\section{The Giants' Shoulders}

Stargazing, in its most primitive form, has entertained our species for hundreds of thousands of years. The ancients used knowledge of the positions of fixed stars as navigational aids and also recognized wandering stars (planets), comets, and novae. The astrolabe, an instrument for fixing the positions of stars, was invented in Greece some 2200 years ago. In medieval times, the positions of stars were of great importance to the Islamic world to indicate the correct times for prayer and the direction of Mecca, and the astrolabe was an important tool. Indeed, one of the greatest observational astronomers, Tycho Brahe, ${ }^{1)}$ performed his measurements without a telescope. He used a "great mural quadrant," whereby stars were sighted through a slot in a wall with a tool attached to a $90^{\circ}$ arc. Accurate measurements of the positions of stars and planets were made in this way, and they remained the most accurate until the introduction of well-built telescopes. Brahe died in 1601, passing his legacy to Johannes Kepler, ${ }^{2}$ who had joined him as an assistant only a year before. Kepler continued to work with Brahe's observations for a decade, during which he developed the laws of planetary motion for which he is most famous, and advanced theories on geometric optics. Kepler was a contemporary of Galileo, ${ }^{3)}$ who is credited with the development and improvement of the refractive telescope. Galileo observed the four largest moons of Jupiter and described the phases of Venus as being similar to the Earth's moon. Hans Lipperhey, ${ }^{4)}$ a German-Dutch lensmaker, is widely considered the inventor of the telescope. However, lenses themselves had been known for at least 2000 years, and are the subject of many writings from ancient and medieval times. Indeed, Ibn al-Haytham, ${ }^{5)}$ considered by many the first scientist, wrote extensively on optical properties of light and vision in his Kitâb al-Manâzir

1) Tyge Ottesen Brahe, 1546-1601, Danish astronomer.

2) 1571-1630, German astronomer and physicist.
3) Galileo Galilei, 1564-1642, Italian physicist, astronomer, and mathematician.

4) $1570-1619$.

5) Abu Ali Hasan Ibn al-Haytham (called Alhazen in Europe), 965-1039, Persian polymath. 
(Book of Optics, 1021), summarized by Roger Bacon over 200 years later in his Perspectiva (1267). Al-Haytham's work was translated into Latin in 1270 as Opticae thesaurus Alhazeni. He described many studies on parabolic and spherical mirrors, the foundation for all modern optical telescopes, and performed the first recorded experiments on the separation of white light into its constituent colors.

Around 1650, in Bologna, Grimaldi ${ }^{6}$ ) observed that small apertures cause what he referred to as diffringere. Moreover, he also observed that diffraction also resulted in coloration. As an astronomer, Grimaldi made accurate maps of the visible geographical features of the Earth's moon and named them after scientists. Grimaldi's diffraction was explained by the wave theory of light put forward by Christiaan Huygens $^{7)}$ in his Traite de la lumière (1690), though the reason of the appearance of different colors eluded him. In addition to studying light, Huygens described the rings of Saturn and the largest of its moons, Titan. The atmosphere of Titan is today the subject of intense chemical research following the successful deployment of the Huygens probe by the Cassini orbiter in 2005.

Isaac Newton ${ }^{8)}$ wrote to the Royal Society in 1672 a letter enclosing his New Theory about Light and Colors:

Where Light is declared to be not Similar or Homogeneal, but consisting of difform rays, some of which are more refrangible than others: And Colors are affirm'd to be not Qualifications of Light, deriv'd from Refractions of natural Bodies, (as 'tis generally believed;) but Original and Connate properties, which in divers rays are divers: Where several Observations and Experiments are alledged to prove the said Theory.

Newton's letter begins, “To perform my late promise to you, I shall without further ceremony acquaint you, that in the beginning of the Year 1666 (at which time I applyed my self to the grinding of Optick glasses of other figures than Spherical), I procured me a Triangular glass-Prisme, to try therewith the celebrated Phænomena of Colours. And in order thereto having darkened my chamber, and made a small hole in my window-shuts, to let in a convenient quantity of the Suns light, I placed my Prisme at his entrance, that it might be thereby refracted to the opposite wall. It was at first a very pleasing divertisement, to view the vivid and intense colours produced thereby."

Newton explained refraction as a property of a corpuscular light, in which the particles of different colors were refracted to different extents. This communication marks the birth of spectroscopy, with the description of color arising from the selective absorption and transmission of various components of the visible spectrum. Newton communicated his studies on light to the general public in his Opticks: or, a Treatise of the Relexions, Refractions, Inflexions and Colours of Light, released in 1704. The treatise was written in contemporary English, rather than Latin, and dealt with

6) Francesco Maria Grimaldi, 1618-1663, Italian mathematician, physicist, and Jesuit priest.

7) 1629-1695, Dutch astronomer, physicist, and mathematician.

8) 1643-1727, English physicist, astronomer, mathematician, and alchemist. 
everyday phenomena in the language of everyday people. Importantly, just like Al-Haytham, Kepler, Galileo, Grimaldi, and Huygens, Newton contributed to the development of astronomy and designed a reflecting telescope that now bears his name. With the telecope and the prism, we have the tools of astronomical spectroscopy. Newton, standing on the shoulders of giants, ${ }^{\text {) }}$ provided the tools with which to investigate phenomena in the greatest laboratory of all: Outer Space.

\section{2}

\section{The First Spectroscopists and Seeds of Quantum Theory}

The wave theory of Huygens and the corpuscular theory of Newton were both hugely successful, yet the wave theory of light gained the upper hand during the nineteenth century. Ironically, Newton's reputation and achievements served as a barrier to further developments in fundamental understanding of light until the late eighteenth century. In November 1801, Thomas Young ${ }^{10)}$ delivered the Bakerian Lecture to the Royal Society entitled “On the Theory of Light and Colours" [1]. In this lecture, he begins carefully by deferring to Newton, going so far as to claim that his theories are mostly a reinterpretation of Newton's own writings: “A more extensive examination of Newton's various writings has shown me, that he was in reality the first that suggested such a theory as I shall endeavour to maintain." Yet, by the end of the lecture, Young has all but destroyed the corpuscular theory of light by demonstrating, quantitatively, that the colors of thin plates, oil films, and diffraction gratings ("Mr Coventry's Exquisite Micrometers") can all be explained by an undulatory theory of light. He postulates that "The Sensation of different Colours depends on the different frequency of Vibrations excited by Light in the Retina," having himself dissected many [2]. Moreover, he accurately calculates the wave number of the extrema of the visible spectrum as $37640 \mathrm{in}^{-1}\left(14820 \mathrm{~cm}^{-1}, 675 \mathrm{~nm}\right)$ and $59750 \mathrm{in}^{-1}\left(23520 \mathrm{~cm}^{-1}, 425 \mathrm{~nm}\right)$, with yellow estimated at $44000 \mathrm{in}^{-1}$ $\left(17320 \mathrm{~cm}^{-1}, 577 \mathrm{~nm}\right)$ (Figure 13.1). William Herschel ${ }^{11)}$ had demonstrated, in 1800 , that the sun's radiation extended into the infrared, and Ritter made similar observations in the ultraviolet the following year. In the same volume of Philosophical Transactions, as Young's lecture is published, Herschel publishes estimates of the sizes of the asteroids Ceres and Pallas [3] and Wollaston ${ }^{12)}$ remarks on dark lines within the solar spectrum [4]. Analyzing the light of a flame, he says that the blue part of the flame exhibits a series of four bands from the red to the blue, and a fifth band at the boundary of the blue and violet regions. The red band was terminated with a

9) Newton used this ancient metaphor in a letter to Robert Hooke, which some have taken as a sarcastic reference to Hooke's stature.

10) 1773-1829, English physician, physicist, and Egyptologist.

11) Friedrich Wilhelm Herschel, 1738-1822, German-born British astronomer. Discov- ered Uranus and named it George, for the king of England.

12) William Hyde Wollaston, 1766-1828, English chemist and physicist. Discovered palladium and rhodium. 


\begin{tabular}{|c|c|c|c|}
\hline Colors. & $\begin{array}{l}\text { Length of at } \\
\text { undulation } \\
\text { in parts of an } \\
\text { inch, in air. }\end{array}$ & $\begin{array}{l}\text { Number of } \\
\text { undulations } \\
\text { in an inch. }\end{array}$ & $\begin{array}{l}\text { Number of undulations } \\
\text { in a second. }\end{array}$ \\
\hline Extreme & .0000266 & 37640 & $46 \mathrm{~g}$ millions of millions \\
\hline Red - - & $.000025^{6}$ & 39180 & 482 \\
\hline Intermediate & .0000246 & 40720 & 501 \\
\hline Orange - - & .0000240 & 4,1610 & 512 \\
\hline Intermediate & .0000235 & 42510 & 523 \\
\hline Yellow － & .0000227 & 44,000 & $54^{2}$ \\
\hline Intermediate & .0000219 & 45600 & $5^{61} \quad\left(=2^{48}\right.$ nearly $)$ \\
\hline Green - - & .0000211 & 47460 & $5^{84}$ \\
\hline Intermediate & .0000203 & 49320 & 607 \\
\hline Blue - & .0000196 & $5^{1110}$ & 629 \\
\hline Intermediate & .0000189 & 52910 & $65^{2}$ \\
\hline Indigo & .0000185 & 54070 & 665 \\
\hline Intermediate & .0000181 & $55^{240}$ & 680 \\
\hline Violet - & .0000174 & 57490 & 707 \\
\hline Extreme - - & .0000167 & $5975^{\circ}$ & 735 \\
\hline
\end{tabular}

Figure 13.1 Young's tabulated wavelengths and wave numbers for visible light, from his 1801 Bakerian Lecture. The frequencies suppose the speed of light to be $500000000000 \mathrm{ft}$. in $8.5 \mathrm{~min}$, $2.99 \times 10^{8} \mathrm{~ms}^{-1}$, from astronomical measurements.

bright line of yellow. On the spectrum of an electrical discharge in air, he says, "I cannot undertake to explain."

Fraunhofer, ${ }^{13)}$ who had become a skilled glassmaker and instrument maker, invented the spectroscope in 1814 . He located 574 dark lines appearing in the solar spectrum, and still denoted Fraunhofer lines in his honor. The most prominent of these dark lines were labeled with letters $A-K$, and Fraunhofer noted the coincidence between the $D$-lines and a yellow emission feature known to occur in flames, which Wollaston had described. In the early 1820 s, he affixed his spectroscope to a telescope in order to study the spectrum of stars other than the sun. The brightest star, Sirius, was found to exhibit a spectrum quite different from and simpler than that of the sun, while that of the Venus was virtually identical, it being seen in reflected sunlight.

Slowly, many of the dark lines exhibited in the solar spectrum were observed in emission from laboratory flames, with various lines becoming associated with particular elements. Indeed, the $D$-lines of sodium observed by Wollaston and Fraunhofer are still referred to as such. The exact explanation for the appearance of lines in absorption and emission came from Kirchhoff and Bunsen ${ }^{14}$ who published, in 1860, a list of laboratory lines and their correspondences with the absorption features in the solar spectrum (Figure 13.2) [5]. Their work conclusively proved that the sun contained the same elements as appeared on earth, among them calcium and sodium. A few years earlier, William Swan ${ }^{15}$ ) described the emission

13) Joseph (von) Fraunhofer, 1787-1826, German.

14) Robert Wilhelm Eberhard Bunsen, 1811-1899, and Gustav Robert Kirchhoff, 1824-1887, German physicists and chemists. Discovered rubidium and caesium.

15) 1818-1894, Scottish. 


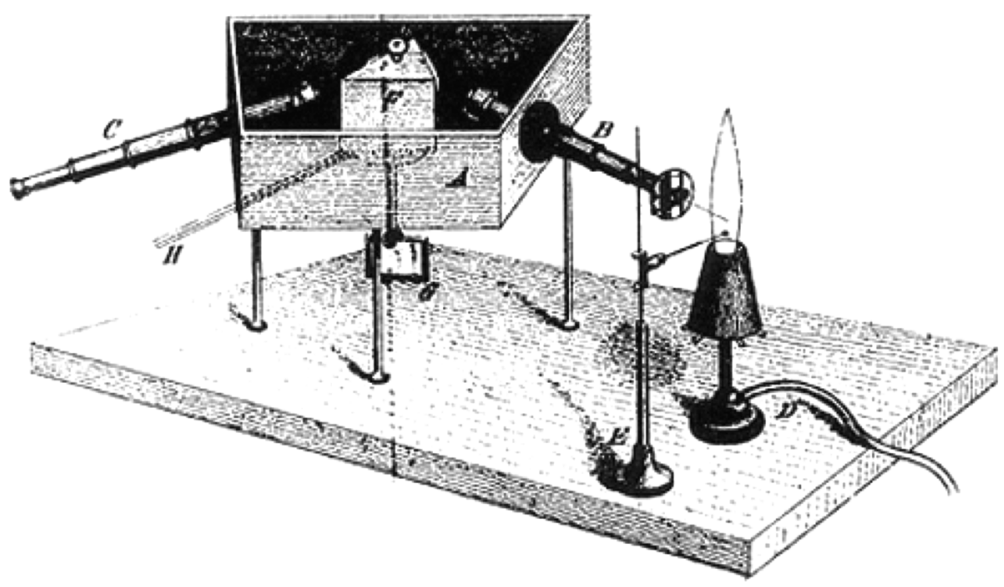

Figure 13.2 Bunsen and Kirchhoff's spectroscope from Annalen der Physik (1860), showing Bunsen's famous burner. With this apparatus, one could observe the Swan bands of $C_{2}$ and the hydrocarbon bands due to the $\mathrm{CH}$ radical.

bands that bear his name to the Royal Society of Edinburgh [6]. These had been observed by Wollaston as the dominant emission from the blue part of a flame.

William Huggins ${ }^{\mathbf{1 6})}$ pounced on the scientific opportunities facilitated by the work of Kirchhoff and Bunsen and published the spectra of many elements in 1864 [7]. He built a telescope and spectrograph in his garden and collected the spectra of various stars, nebulae, and comets. This work was published almost simultaneously with that of the American L.M. Rutherfurd, who also collected and classified various stellar spectra. The first cometary spectrum, of Comet Tempel, was reported in 1864 by Donati, ${ }^{17)}$ who reported the appearance of the Swan bands [8]. The appearance of Swan bands in cometary spectra was confirmed by Huggins in 1868 in his report of the spectrum of Comet Winnecke and also in Coggia's Comet of 1874 [9, 10]. Remarkably, by comparison with the positions of Swan bands obtained from a terrestrial flame, Huggins demonstrated that the relative velocity of the comet was some 40 miles per second. The bright band near Fraunhofer's G-line at $4300 \AA$ was conspicuously absent from the comet's spectrum, being normally associated with the Swan bands.

The Great Comet of 1881 was first observed by Tebbutt at Windsor, New South Wales, now on the outskirts of Sydney, ${ }^{18)}$ and its spectrum was obtained by Huggins and others (Figure 13.3) [11]. Huggins correctly concluded that the bright lines appearing at 3883 and $3870 \AA$ were due to carbon, in combination with nitrogen, noting Liveing and Dewar's demonstration that the spectrum was obtained from cyanogen [12]. Huggin's 1881 spectrum also revealed a band near $4050 \AA$, which would not be identified for another 70 years.

16) 1824-1910, English astronomer.

17) Giovanni Battista Donati, 1826-1873, Italian astronomer.

18) John Tebbutt, 1834-1916, Australian astronomer. 

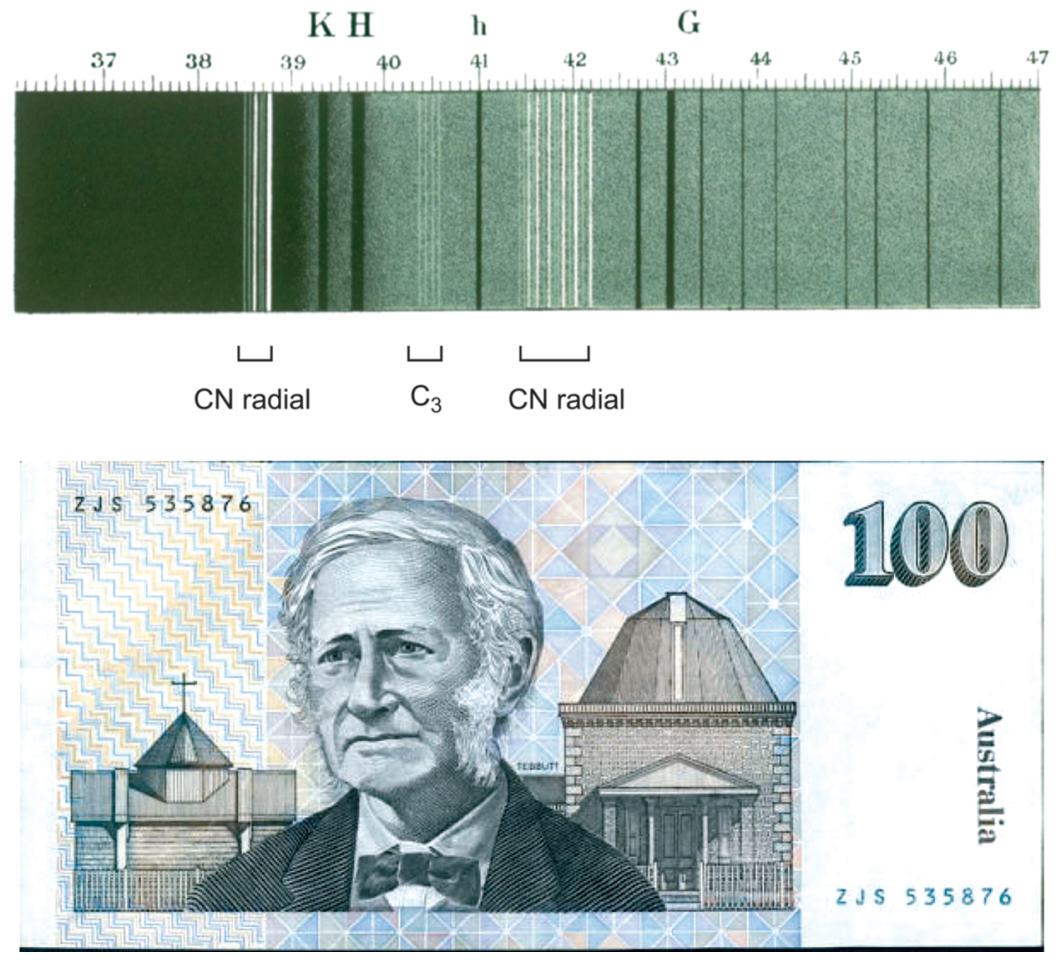

Figure 13.3 Top: The spectrum of the Great Comet of 1881, discovered by Tebbutt, as recorded and published by Huggins. The Fraunhofer lines indicated are due to $\mathrm{Ca}^{+}(\mathrm{H}$, $K)$, Fe (G), and $\mathrm{H} n=5 \rightarrow 2(h)$. Two molecular species are responsible for the emissions, $\mathrm{CN}$ and $C_{3}$. The latter would not be identified until 1951. Bottom: Tebbutt and his observatories as depicted in Australia's original $\$ 100$ note.

By the late nineteenth century, spectroscopy was established as an astronomical tool of great utility. The inherent curiosity of our species had spurred fundamental studies of nature that had by now revealed the spectra of elements and molecules, though these were not yet identified as such. The comets had revealed the great ubiquity of the carbonaceous Swan bands, a hydrocarbon-associated emission of about $4300 \AA$, and bright emissions from a cyanogen-related form of carbon. These phenomena were revealed to fundamental physicists by astronomical spectroscopists, but a proper understanding of them would have to wait until the advent of quantum mechanics.

This chapter is about the relationship between astronomical molecular spectroscopy and computational spectrometry. A brief account of how the former gave rise to the discovery of the quantum mechanical phenomena that necessitated the latter has already been given. Happily, in 2010, we are approaching the age whereby quantum mechanics can "give back" to astronomy and assist in solving problems, some as old as 90 years. Between these periods, molecular astronomy and computational spectrometry have walked hand in hand, one aiding the other in development and 
interpretation. In the following sections, we will deal with molecular astronomy across four orders of magnitude of the electromagnetic spectrum, using case studies to illustrate how computational spectrometry has advanced since the beginning of the quantum theory.

\section{3}

\section{Small Molecules}

The spectra of many small molecules were obtained astronomically in the late nineteenth and early twentieth centuries. However, in many instances, their identification depended on the detailed understanding of spectroscopy afforded by the developments in quantum theory in the first few decades of the twentieth century. As of 2010, spectroscopic constants of small astronomical molecules can be calculated to within $0.1 \%$, but it has not always been so. Nevertheless, computational spectrometry has played its part in interpreting astronomical spectra throughout the twentieth century, and the exquisite data afforded by molecular spectroscopy have provided quantum chemists with the sparring partners necessary to hone their code.

\subsection{1}

\section{$\mathrm{CH}, \mathrm{CN}, \mathrm{CO}, \mathrm{CO}^{+}$}

By the end of the nineteenth century, the hydrocarbon spectrum observed by Wollaston had been extensively reproduced in the laboratory. Although it was not possible to establish with certainty whether hydrogen was required for production of the Swan bands at 5635, 5165, and $4737 \AA$, it appeared that it was required for the 4315 A band, and as such this and the other bands associated with this feature came to be known as the "hydrocarbon bands." Similarly, the "cyanogen bands," identified first in comets, were known to involve both carbon and nitrogen. Liveing and Dewar referred to these as the nitrocarbon bands in 1880 [12], and they were observed brightly in the Great Comet of 1881 [11]. Other band systems known to be associated with carbon were the Angström bands [13] and the "comet tail bands" [14].

The comet tail bands were first observed in the spectra of comets Daniel (1907d) and Morehouse (1908c), especially bright in the tails. They were reproduced by Fowler and shown most likely to be due to $\mathrm{CO}[14,15]$. As they were always produced at very low pressures, some referred to these spectra as the low-pressure carbon bands. While CO is a major component of cometary gas, the lowest transition that can be excited from the ground state is already in the vacuum ultraviolet and inaccessible to early astronomers. As such, the spectrum of neutral CO was not known at first by cometary spectra but by experimentation with discharge tubes.

Birge noted in 1926, "Until very recently it was impossible to give the exact chemical origin of practically any band system, even of those most exhaustively investigated. The long controversy concerning the Swan bands is a case in point." Indeed, the origin of the Swan bands in $\mathrm{C}_{2}$ would not be accepted for a few years, and Birge thought "it is doubtful such a molecule (diatomic) exists" [16]. In 1926, 
The National Research Council (Washington) published "Molecular Spectra in Gases," the report of the National Research Council Committee on Radiation in Gases. A bound copy would set you back $\$ 4.50$, but it would have been worth it just to read Chapter 2: "a thirty page survey of 'quantum dynamics and the correspondence principle', ... entirely on the basis of the old quantum theory rather than the Heisenberg-Schroedinger mechanics, which were developed too late for incorporation" [17]. Notwithstanding, the theories were advanced sufficiently that moments of inertia extracted from the quantized rotational structure were sufficient to identify the carriers of the hydrocarbon and cyanogen bands as $\mathrm{CH}$ and $\mathrm{CN}$, respectively. The identification of the comet tail bands with $\mathrm{CO}^{+}$stems from a combination of the chemical evidence, the measured spectroscopic constants (now more or less understood), and the fact that they occur in the region of the discharge tube associated with cations [16]. These three diatomic molecules were among the first radicals to be identified. They were discovered in space and contributed to the development of the spectroscopy, which in the mid-1920s was developing into a quantitative science.

In 1940, MacKellar reported on "Evidence for the molecular origin of some hitherto unidentified interstellar lines" [18]. Coincidences between the lowest $J$-lines of $\mathrm{CH}$ and $\mathrm{CN}$ demonstrated these species to be interstellar. Indeed, from these spectra, the temperature of interstellar space is calculated to not to exceed $2.7 \mathrm{~K}$. In fact, this is also the first evidence for the cosmic microwave background (CMB), for the discovery of which, in 1965, Penzias and Wilson were awarded the 1978 Nobel Prize in Physics. The dipolar CN molecule has since been used as a molecular thermometer of the CMB [19]. In order to calculate column densities of $\mathrm{CN}$, the oscillator strengths must be known. The oscillator strengths in use are those of Roth, Meyer, and Hawkins [20], who calculated values from a combination of total electronic oscillator strength (from Jackson's laser excitation studies [21]), FranckCondon and Hönl-London factors.

Cartwright and Hay, at Los Alamos, made an attempt to calculate oscillator strengths for the CN violet and red systems in 1982 [22]. Their CI study utilized a set of orbitals derived from an MCSCF calculation of the anion, which is isoelectronic with $\mathrm{N}_{2}$. With a [ $4 \mathrm{~s} 3 \mathrm{p} 1 \mathrm{~d}$ ] basis, a decent agreement was found between the measured and the calculated fluorescence lifetimes for the $B^{2} \Sigma^{+}$state (violet system), with the calculated values about $6 \%$ high. However, the calculations for the $A^{2} \Pi \rightarrow X^{2} \Sigma^{+}$red system were not in agreement with the experiment. Sumner Davis et al. at Berkeley sought to clarify the relative oscillator strengths of the violet and red systems in 1986, after discussions with Cartwright and Hay [23]. They directly compared the oscillator strengths of the red and violet systems, and calibrated their results with a check of the fluorescence lifetime of the $v^{\prime}=0$ level of the $B^{2} \Sigma^{+}$state. The results confirmed the validity of the experimental findings, and prompted theorists at NASA to reattempt the calculation of the CN red system. In 1988, Bauschlicher, Langhoff, and Taylor calculated oscillator strengths and emission lifetimes for the red and violet CN systems [24]. They performed MRCI calculations with a $[5 \mathrm{~s} 4 \mathrm{p} 3 \mathrm{~d} 2 \mathrm{f} 1 \mathrm{~g}]$ basis, calculating the radiative lifetime of the $v^{\prime}=0$ level of the violet system in accord with that measured by Davis, but a little lower than some 
earlier investigations. Moreover, their calculated lifetimes for the violet system were nearly identical with those of Cartwright and Hay. Again, the calculated lifetimes for the red system were about twice as long as the experimental values. Lu, Huang, and Halpern remeasured the vibrational levels of the red system in 1992, confirming the error in the calculated lifetimes [25]. They suggested that the calculations had omitted some effects assumed to be small, such as rotational interactions or quadrupole terms. So, while astronomers can be satisfied that the experimental oscillator strengths are reliable, it remains for computational spectrometry to predict the correct lifetimes for the $\mathrm{CN}$ red system.

\subsection{2}

\section{Dicarbon: $\mathrm{C}_{2}$}

The Swan bands were known to be associated with carbon, but there persisted uncertainty as to the carrier as late as 1927 [26]. Johnson concludes, "The evidence both of direct experiment and of analysis is conclusive in assigning the Swan bands to a HC-CH molecule." The same year Mulliken argued that the Swan bands are "probably $\mathrm{C}_{2}$ " [27], and by 1929 Johnson had come around to this way of thinking, influenced, in part, by "more especially the rapid theoretical developments due to the work of Mulliken and others" [28]. This paper also revealed more information on Fowler's "high-pressure bands of carbon" $[14,15]$, which were shown to share the lower state with the Swan bands. ${ }^{19)}$ In the same paper, Fowler described reproduction of the "tail bands" of comets in low-pressure CO, alluding to conversations with Huggins that suggested that it was these bands that were seen in the 1868 comet (Bronson). In 1939, Mulliken predicted 33 electronic states of $\mathrm{C}_{2}$, and calculated bond lengths with remarkable accuracy using semiempirical formulas honed on the knowledge of $\mathrm{CN}, \mathrm{N}_{2}{ }^{+}, \mathrm{N}_{2}$, and $\mathrm{O}_{2}$ [29]. It was unknown then whether the triplet ${ }^{3} \Pi_{u}$ or singlet ${ }^{1} \Sigma_{g}^{+}$state should be the "normal" state of $\mathrm{C}_{2}$, with Mulliken leaning toward the triplet, without ruling out other possibilities, including ${ }^{3} \Sigma_{g}^{-}$. These issues were resolved by the discovery of a band system by Ballik and Ramsay in 1958 that shared the lower state with the Swan and Fox-Herzberg systems [30]. This had, as its upper level, the ${ }^{3} \Sigma_{g}^{-}$state that Mulliken had entertained as a possible ground state. Moreover, perturbations in the ${ }^{3} \Sigma_{g}^{-}$levels were found to match equal and opposite perturbations in the ${ }^{1} \Sigma_{g}^{+}$state, revealing the singlet state as the ground state of $\mathrm{C}_{2}$. In a conversation between Ramsay and the author, ${ }^{20}$ ) Ramsay described that Herzberg took as many as eight matching perturbations as sufficient evidence as to this now established fact. The band systems of greatest astrophysical importance then known were the $d^{3} \Pi_{g} \rightarrow a^{3} \Pi_{u}$ Swan, $e^{3} \Pi_{g} \rightarrow a^{3} \Pi_{u}$ Fox-Herzberg, $b^{3} \Sigma_{g}^{-} \rightarrow a^{3} \Pi_{u}$ Ballik-Ramsay, $D^{1} \Sigma_{u}^{-} \rightarrow X^{1} \Sigma_{g}^{+}$Mulliken, and $A^{1} \Pi_{u} \rightarrow X^{1} \Sigma_{g}^{+}$Phillips systems. The $c^{3} \Sigma_{u}^{+}$ state predicted by Mulliken could not be identified in spectra until the next century (Figure 13.4).

19) These bands were, in fact, shown by Herzberg to be Swan bands with $v^{\prime}=6$, this state being excited selectively from the recombination of two carbon atoms in a three-body collision.

20) International Symposium on Free Radicals, Big Sky, Montana, 2007. 


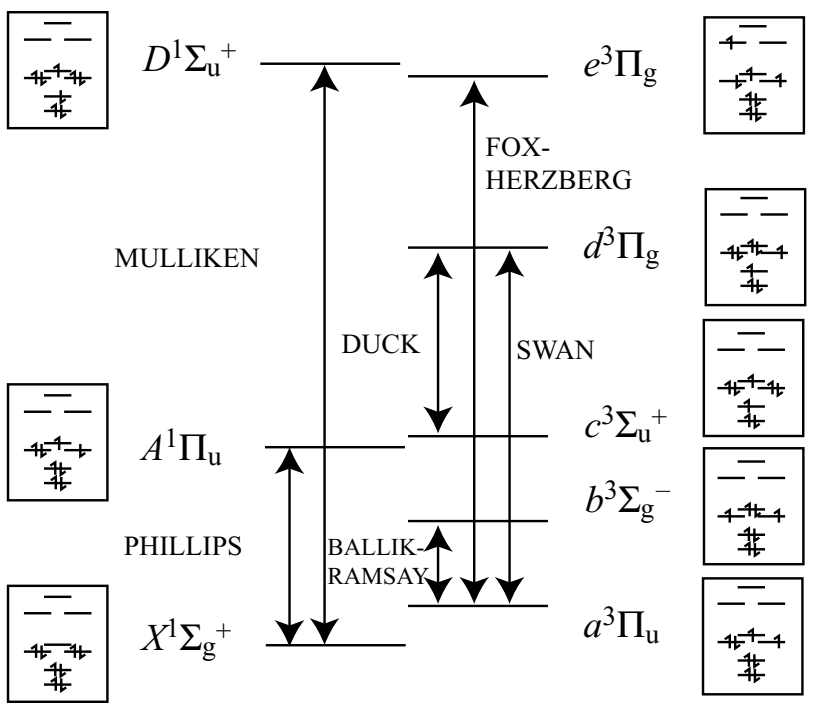

Figure 13.4 Some of the valence states of $C_{2}$, with notable band systems indicated. The "Duck" bands were discovered in the author's laboratory in 2006, the name being coined by Klaas Nauta for $d-c$, and the proximity of a pub called the Duck and Swan (not to scale).

With the ground state of $\mathrm{C}_{2}$ fixed, and the positions of several states known, Clementi proceeded to calculate the oscillator strengths of several band systems of $\mathrm{C}_{2}$ with impressive accuracy [31], using the "magic formula" of Mulliken to calculate the hybridization of the molecular orbitals [32], checked against LCAO-MO calculations. Clementi used a compromise between his calculated values and the contemporary experimental values for the oscillator strength of the Swan system to estimate the abundance of dicarbon in the solar reversing layer, the Swan bands having been observed in absorption in the spectrum of the sun [33].

In 1977, Souza and Lutz confirmed the dicarbon molecule as a component of the interstellar medium, as evidenced by Phillips band absorption in the line of the sight toward Cyg OB2 No. 12 [34]. This was the first new molecule identified in the interstellar medium by optical spectroscopy since the late 1930s. In arriving at a total abundance in this line of sight of $1 \times 10^{14} \mathrm{~cm}^{-2}$, they used the experimentally determined oscillator strength for the 1-0 band of $2.4 \times 10^{-3}$ from Roux [35], which did not differ so much from the earlier calculated result of Clementi. The following year Chaffee and Lutz observed dicarbon toward $\zeta$-Ophiuchi, reporting a total column density of $7.9 \times 10^{12} \mathrm{~cm}^{-2}$ [36].

At the same time, with these astronomical developments, there were several attempts to conquer $\mathrm{C}_{2}$ by theory. Arnold and Langhoff published their CI study of the Swan system in 1978 [37], and the same year a similar study by Zeitz et al. was also published [38]. Chabalowski et al. improved upon these calculations in 1981 [39], reporting accurate oscillator strengths for the Swan and Fox-Herzberg systems, and reported the results of similar calculations on the Mulliken, Phillips, and Ballik-Ramsay systems in 1983 [40]. These calculations are in very good agreement 
with the most recent ones from the author's research group in Sydney. The motivation for undertaking these calculations in Sydney is an interesting story and highlights how computational spectrometry is now so advanced as to precede experiment.

In 2004, Robert Sharp (Anglo-Australian Observatory) and the author undertook observations on the Red Rectangle (q.v.). It was pointed out to us by Robert Glinski (Tennessee Tech.) that our spectra of the $5800 \AA$ Red Rectangle bands (RRBs) had also captured the $(0,1)$ Swan band of $\mathrm{C}_{2}$. The Swan bands are dwarfed by the RRBs for which the carrier is unknown. Nevertheless, we undertook to model the $\mathrm{C}_{2}$ photophysically, on the initial assumption that $\mathrm{C}_{2}$ was a "solved problem." This sort of kinetic model had been built before by various groups [41]. However, there appeared to be some uncertainty in the transition moments of some of the band systems invoked in the models, which normally included the $a^{3} \Pi_{u}, b^{3} \Sigma_{g}^{-}, c^{3} \Sigma_{u}^{+}, d^{3} \Pi_{g}, X^{1} \Sigma_{g}^{+}$, and $A^{1} \Pi_{u}$ states. I approached my friend and colleague, George Bacskay, to calculate these states using the highest level possible in 2006. Partitioning the work between us and a graduate student, Damian Kokkin, we obtained, eventually, excellent results for the primary spectroscopic constants, generally to within $0.1 \%$. This triumph was confused only by the terrible agreement with the literature for the $c^{3} \Sigma_{u}^{+}$state, where $\omega_{\mathrm{e}}$ was calculated at $2061 \mathrm{~cm}^{-1}$ with the experiment reporting $2085 \mathrm{~cm}^{-1}$ [42]. Such an agreement might have been satisfactory 20 years earlier, but since all other states agreed with the reported spectroscopy to within $0.1 \%$, we were confident that the calculations for the $c^{3} \Sigma_{u}^{+}$state were indeed accurate, and searched the literature thoroughly. As it turned out, the $c^{3} \Sigma_{u}^{+}$state had actually never been observed, except in a photodetachment spectrum of the anion [43]. The reported constants were the result of fitting five spectroscopic constants to five perturbations of the $c^{3} \Sigma_{u}^{+}$state with the $A^{1} \Pi_{u}$ state [44].

Being ambitious spectroscopists, and with the tools at our disposal, we undertook a laboratory search for the predicted $d^{3} \Pi_{g} \leftarrow c^{3} \Sigma_{u}^{+}$fluorescence excitation spectrum, monitoring Swan emission, which theory had shown would dominate the Einstein $A$ coefficient. Due to the metastable nature of the $c^{3} \Sigma_{u}^{+}$state, it having no symmetry or spin allowed downward transitions, it is formed easily enough (as it turned out) in a pulsed electric discharge through argon containing about $1 \%$ acetylene. We obtained excellent signal on the first day of trying and after some weeks had extracted spectra of sufficient quality to extract the spectroscopic constants predicted by our calculations $[45,46]$. The experiments confirmed the validity of the computational results, with $\omega_{\mathrm{e}}$ measured at $2061.940 \mathrm{~cm}^{-1}$, only $0.03 \%$ above the calculated value. This example of computational spectrometry taking priority of experiment, while seemingly minor, gives hope for the future, when age-old problems of astronomical spectroscopy may be solved using computers.

\subsection{3}

\section{The Carbon Trimer: $\mathrm{C}_{3}$}

What appear to be four lines centered around $4050 \AA$ in Huggin's spectrum of the Great Comet of 1881 would prove a mystery for 70 years [11]. The bands appeared in many comets, though not all, sometimes when the $\mathrm{CH}$ spectrum around $4300 \AA$ a was 
absent. In her report of the study of the spectrum of Comet Mellish (1915), Glancy notes the appearance of the 4050 A group, which had also been observed in Comets Zlatinsky (1914) and Brooks (1910) [47]. Importantly, she connects these bands with the 1916 observations of Raffety in his study on discharges with carbon electrodes [48]. Indeed, Raffety's work appears to be the first laboratory detection of the 4050 A group, and these bands were denoted Raffety's Bands by Bobrovnikoff in his 1931 review, in which he suggests $\mathrm{CN}$ as the carrier of the bands [49]. Swings et al. discussing the appearance of the bands in Comet Cunningham (1940), regard the use of the term "Raffety's Bands" inadequate and suggest the carrier to be an as yet uninvestigated polyatomic molecule [50]. In 2010, the bands were commonly referred to as the "comet bands" for obvious reasons. The comet bands were reproduced by Herzberg in the laboratory in 1942 [51]. He suggested $\mathrm{CH}_{2}$ as the carrier, but it was shown by Monfils and Rosen, using a deuterated precursor, that the carrier possessed no hydrogens [52]. However, suggesting nothing else, the identification remained a mystery until 1951, with a landmark paper by Douglas [53]. Comparing laboratory spectra obtained with natural and ${ }^{13} \mathrm{C}$-enriched precursors, Douglas concluded that the carrier was most likely $\mathrm{C}_{3}$, which was confirmed by a follow-up study in 1954 [54]. The spectroscopy of $C_{3}$ was analyzed in detail in 1965 by Gausset et al., a paper which, at the time of writing, has been cited nearly 250 times [55].

The carbon trimer poses a tricky problem for computational spectrometry, the upper state being plagued by the complications of Renner-Teller coupling. The ground state is also rather unusual, having a vibrational frequency of only $63.4 \mathrm{~cm}^{-1}$. Liskow et al. were able to calculate this at $69 \mathrm{~cm}^{-1}$ in 1972 [56], but this was perhaps fortuitous as very high-quality calculations by Saha and Western in 2006 yielded $85 \mathrm{~cm}^{-1}$ for this mode [57]. In 1977, Perić-Radić et al. in Bonn treated both the ground and the excited states of $\mathrm{C}_{3}$ with configuration interaction using a polarized doublezeta basis [58]. They obtained excellent agreement with the excited state position, predicting a value only $0.03 \mathrm{eV}$ too low, or $4 \mathrm{~nm}$ to the red. However, the calculated ground-state bending frequency is quite poor. They calculated the total oscillator strength for the $A^{1} \Pi_{u} \leftarrow X^{1} \Sigma_{\mathrm{g}}^{+}$transition to be about $f=0.061$. Another result reported is the theoretical position of the lowest triplet state of $\mathrm{C}_{3}$, which Perić-Radić and coworkers put at $2.04 \mathrm{eV}$ seemingly confirming the $2.10 \mathrm{eV}$ phosphorescence observed in matrix isolation spectra as originating in the $a^{3} \Pi_{u}$ state. The precise position of this state is not known from gas-phase spectroscopy, and may be astronomically interesting. Chabalowski et al. improved on the previous results of the Bonn group in 1986 with a study employing a much larger basis set, revising the oscillator strength to $f=0.052$ [59]. This would correspond to an observable emission lifetime of about $95 \mathrm{~ns}$ for the $A^{1} \Pi_{u} \rightarrow X^{1} \Sigma_{\mathrm{g}}^{+}$transition.

In 2001, Maier and coworkers succeeded in observing $C_{3}$ in absorption toward a number of astronomical sources, namely, $\zeta$-Ophiuchi, 20 Aquilae, and $\zeta$-Persei (Figure 13.5) [60]. In calculating the column densities of $C_{3}$ toward these sources, Maier et al. opted for a value of $f=0.016$, some three times smaller than the ab initio result of Chabalowski et al. Now, the theoretical result is a total oscillator strength, assuming a Franck-Condon factor of unity. Nevertheless, the predicted emission 
Simulation $(80 \mathrm{~K})$

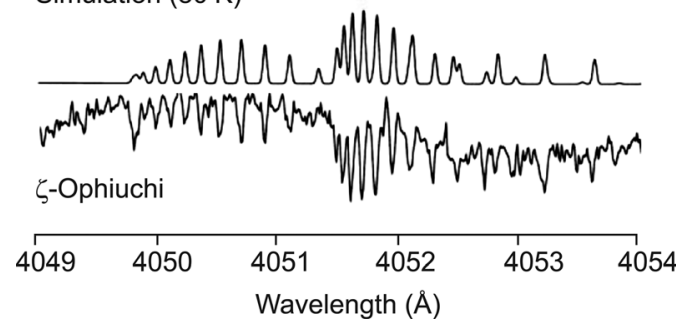

Figure 13.5 The absorption spectrum of $\mathrm{C}_{3}$ seen toward $\zeta$-Ophiuchi by Maier et al. [60] compared with a calculated spectrum at $80 \mathrm{~K}$.

lifetime is directly comparable with observations. Indeed, the oscillator strength of $\mathrm{C}_{3}$ is calculated from emission lifetimes on the order of $200 \mathrm{~ns}$ observed by Becker et al. in 1979 [61]. A recent reinvestigation by Zhang et al. of the lifetimes of rovibrational lines of $\mathrm{C}_{3}$, in 2005, shows Becker and coworkers' experimental results to be essentially correct [62]. Their reported emission lifetimes span 192-227 ns for low $J$-levels of the $A^{1} \Pi_{u}(000)$ state. The discrepancy between these values and those predicted $a b$ initio is possibly due to the variation of the transition moment with geometry. The theoretical value of Chabalowski et al. is a $f_{\mathrm{e}}$ value, evaluated only at the equilibrium geometry. However, a 2002 MR-AQCC calculation quotes a value of $f_{\mathrm{e}}=0.02$, in accord with the derived experimental total oscillator strength of 0.0246 [63]. Although the 2006 results of Saha and Western are generally excellent, they do not report oscillator strengths [57]. It would appear that the defining computational spectrometry for $\mathrm{C}_{3}$ is yet to be reported. $\mathrm{C}_{3}$ has now been observed in many translucent sight lines toward reddened stars [64] and in the infrared spectrum of the molecule factory, IRC +10216 , and Saggitarius B2 $[65,66]$. As a fundamental component of the interstellar medium, accurate calculations on the spectrosocpy of $\mathrm{C}_{3}$ will certainly illuminate its role in the chemistry of space.

\subsection{4}

\section{Radioastronomy}

In the mid-1930s, it was shown by Cleeton and Williams that gases could absorb microwaves in the centimeter region [67]. Following the World War II, scientists who had diverted their attention to the development of radar could redirect their efforts to fundamental science. In 1946, there was an explosion of spectroscopy of water and ammonia in the centimeter region $[68,69]$. However, detection of ammonia and then water emission from extraterrestrial sources did not come until the reports of Cheung et al. in the late 1960s [70, 71]. Cheung's coauthors included Charles Townes, who had won the Nobel Prize in Physics in 1964 "for fundamental work in the field of quantum electronics, which has led to the construction of oscillators and amplifiers based on the maser-laser principle." These reports were the first of the existence of polyatomic molecules in the interstellar regions, as opposed to transient species in comet tails. While the first molecules in space were identified through electronic spectroscopy, it was the radioregions that yielded, by far, the majority of interstellar 
molecular identifications. As of July 2000, there were 123 interstellar molecules known, with new discoveries being added at a rate of about 4 per year [72]. In molecular radioastronomy, line positions can be measured to a precision of 1 part in $10^{7}$, with laboratory measurements matching this precision [73]. Since rotational spectroscopy is a ground-state phenomenon, it poses excellent challenges and opportunities to various computational methods. As radioastronomy progressed, many new unidentified lines, or " $U$ " lines, were discovered. The discovery of the identity of these interstellar molecules is greatly enhanced by theoretical predictions of structure and centrifugal distortion. An excellent example of this is the first detection of an interstellar anion. In 1995, Kawaguchi and coworkers reported a series of harmonically related U-lines, a signature of a linear molecule, in a survey toward IRC +10216 , a source often called the "molecule factory" [74]. Seven lines were identified consistent with a linear molecule having a rotational constant of 1376.8641(4) MHz. From this, the unidentified species was designated B1377. Aoki performed ab initio calculations of various candidates [75]. He noted, in 2000, that the rotational constant was similar to $\mathrm{C}_{6} \mathrm{H}$ and $\mathrm{C}_{5} \mathrm{~N}$, and while noting that no anion had yet been discovered in interstellar space, recommended that "the U-lines with the rotational constant of $1377 \mathrm{MHz}$ may originate from the $\mathrm{C}_{6} \mathrm{H}^{-}$anion. The $\mathrm{B} 1377$ spectrum was reproduced in the laboratory in 2006 by McCarthy et al. at the HarvardSmithsonian Center for Astrophysics [76]. The laboratory B-constant was measured at 1376.86298 (7) $\mathrm{MHz}$, compared to the astronomical measurement, 1376.86248 (294) MHz. John Stanton performed a CCSD(T)/cc-pVTZ calculation to predict a rotational constant of $1376.9 \mathrm{MHz}$, confirming without doubt the chemical origin of the $\mathrm{B} 1377$ spectrum. Consequently, $\mathrm{C}_{4} \mathrm{H}^{-}$was discovered in the envelope of the carbon star IRC +10216 [77]. The anion had been previously identified, along with $\mathrm{C}_{8} \mathrm{H}^{-}$, in the laboratory by the Harvard-Smithsonian group [78]. They compared measured constants to Stanton's CCSD(T)/cc-pVTZ calculations with the vibrationrotation correction calculated at the $\operatorname{CCSD}(\mathrm{T}) / \mathrm{cc}-\mathrm{pVDZ}$ level of theory. For both anions, the error is only $0.02 \%$, highlighting the importance and success of computational spectrometry in aiding the identification of laboratory and interstellar spectra. $\mathrm{C}_{8} \mathrm{H}^{-}$has since been detected in the Galactic molecular source TMC-1 [79].

\section{4}

\section{The Diffuse Interstellar Bands}

The first descriptions, in 1922, of unidentified absorption features seen toward reddened stars were due to Mary Lea Heger [80]. Merrill's studies had demonstrated that these features did not follow the oscillatory motion when observed toward binary systems and thus were due to the interstellar medium [81]. Merrill knew of four features, at 5780.4, 5796.9, 6283.9, and 6613.9 A with a vague feature at $4427 \AA$ A being "suspected." There are now hundreds of confirmed diffuse interstellar bands, but not a single one has been assigned to a carrier, despite decades of detailed investigation. In the late 1970s, it was generally assumed that the carriers arose from impurities in grains acting as color centers. However, Smith et al. renewed interest in molecular 
carriers [82]. Also in 1977, Douglas, who had years earlier identified $\mathrm{C}_{3}$ in the laboratory, suggested carbon chains as carriers of the diffuse interstellar bands and that the observed widths of the lines arose from the rapid internal conversion [83]. It was known by then from radioastronomy that carbon chains existed in the interstellar medium.

Computational results aid in interpreting and understanding laboratory spectra. While calculations may not always be able to reproduce oscillator strengths and band positions quantitatively, there is still value in qualitative results. Coulson would have agreed. At the molecular quantum mechanics conference in 1960 at Boulder, Colorado, he is said to have pleaded in his after dinner speech "give us insight, not numbers!" [84]. An example of this with regard to carbon chain molecules and DIBs is in the strengths of electronic transitions of odd, hydrogen-terminated carbon chains, $\mathrm{HC}_{2 n+1} \mathrm{H}$ [85]. These molecules possess one $\pi_{x}$ and one $\pi_{y}$ electron for each carbon in the chain, ensuring that the ground state has odd occupancies of each of the two perpendicular $\pi$-systems. The ground state is thus a triplet, and electronic excitations take place by promoting electrons from the $n$th $\pi_{x / \gamma}$ orbital to the $n+1$ th, or from the singly occupied $n+1$ th to the $n+2$ th (LUMO). At the Hückel level, these transitions give rise to a pair of degenerate excited states, due to the symmetry of the energy-level spacings in the $\pi$-system. Introducing configuration interaction acts to split these states into even and odd combinations of the one-electron excitations. As it turns out, the lower energy excited state, which had been the focus of laboratory investigations, is the odd combination [86]. This has the consequence that the transition moments of each one-electron excitation cancel, and the $A^{3} \Sigma_{u}^{-} \leftarrow X^{3} \Sigma_{g}^{-}$ carries very little oscillator strength and is therefore not likely to be a good candidate for astronomical detection (a match to a DIB would have been self-evident). The oscillator strength is carried by the $B^{3} \Sigma_{u}^{-} \leftarrow X^{3} \Sigma_{g}^{-}$transition, which we calculated by CASSCF to have oscillator strengths greater than unity and increasing with chain length. For $\mathrm{HC}_{19} \mathrm{H}$, which we detected using R2C2PI spectroscopy, the CASSCF $B^{3} \Sigma_{u}^{-} \leftarrow X^{3} \Sigma_{g}^{-}$oscillator strength is calculated to be about 10 . Higher level calculations by Mühlhäuser et al. using MRCI for smaller members of the series predict an f-value about two-thirds this value [87]. Indeed, these consequences are predicted by the pairing theorem put forward by Coulson and Rushbrooke in 1940 [88].

Observations on infrared emissions at characteristic wavelengths in the 1970s led to polycyclic aromatic hydrocarbons (PAHs) being suggested as the carrier [89]. PAHs are transiently heated by absorption of ultraviolet photons and then fluoresce in the infrared through vibrational transitions on the ground state following internal conversion. Léger and d'Hendecourt dedicated their 1985 paper on the DIBs to the memory of Douglas, in which they hypothesize that the DIBs are due to polycyclic aromatic hydrocarbons [90]. A similar hypothesis was put forward by van der Zwet and Allamandola [91]. Crawford, Tielens, and Allamandola, realizing that even small PAH cations exhibited transitions in the DIB range, proposed this class of molecular ion as a set of candidates [92]. With carbon chains and PAHs, neutral or ionized, we have the most seriously considered candidates for the DIB carriers. Computational spectroscopy may be of great use to experimentalists in identifying candidate carriers for investigation in the laboratory. For instance, for PAHs comprising between 4 and 
10 fused benzene rings, there are over 20000 possibilities! It is clearly impossible to synthesize every possible species and measure its excitation under simulated astrophysical conditions.

Weisman et al. applied TDDFT with the $6-31 G^{*}$ basis to show that closed shell PAH cations exhibit spectra dominated by a strong absorption in the DIB range, with oscillator strengths in the $f \approx 0.2$ range [93]. Since few DIBs exhibit any perfect correlation in equivalent width, it is generally assumed that each DIB arises from a different species, hence the need for a single strong transition to dominate the spectroscopy. Recently, Hammonds et al. performed a similar study on hydrogenated and protonated PAHs [94]. For more standard PAHs and their cations, a database is maintained by Malloci et al. at http://astrochemistry.ca.astro.it/database/. At the time of writing, it contained calculated spectra of 40 PAHs in various charge states.

In 2010, with the DIB problem still unresolved, the problem lies more with experimental techniques. It is rather simple these days to select a candidate molecule, optimize the geometry at the $\mathrm{B} 3 \mathrm{LYP} / 6-31 \mathrm{G}^{*}$ or a similar level, and run a TDDFT calculation. The positions of excited states may be within $0.3 \mathrm{eV}$ of the eventual answer, but matches to DIBs must be spectroscopically exact. In the author's opinion, a real contribution to solving the problem may come from finding, using theoretical methods, a chromophore with large oscillator strengths and near-vertical excitations. In doing so, laboratory measurements may be guided toward more likely DIB carriers and effort will be more fruitfully spent.

\subsection{1}

\section{The Hump}

The most prominent feature on the interstellar extinction curve, and thus the biggest DIB of all, is the so-called $2175 \AA$ "hump," discovered in 1965 [95]. It was immediately realized that this could be explained by graphitic particles in the interstellar medium, an idea that fits well with the later proposed carriers of the DIBs and the AIBs. In 1991, using the CNDO/S method, Braga et al. suggested that fullerenes could be responsible for the hump [96]. Sitting between fullerenes and graphitic particles, carbon onions were proposed in 2003, from comparisons with actual spectra [97]. Recent calculations at the B3LYP/4-31G level have shown that a mixture of dehydrogenated PAHs could give rise to this feature $[98,99]$. So, it would appear that there is a range of materials that could conspire together to give rise to the $2175 \AA$ hump. Unfortunately, computational spectrometry is not yet at a stage to rule out candidates, but rather shows that a great variety of aromatic carbonaceous materials may be responsible.

\section{5}

\section{The Red Rectangle, HD44179}

The Red Rectangle is one of the brightest objects in the sky when viewed at a wavelength of $3.3 \mu \mathrm{m}$ (Figure 13.6) [100]. It has a peculiar shape, which at low resolution 


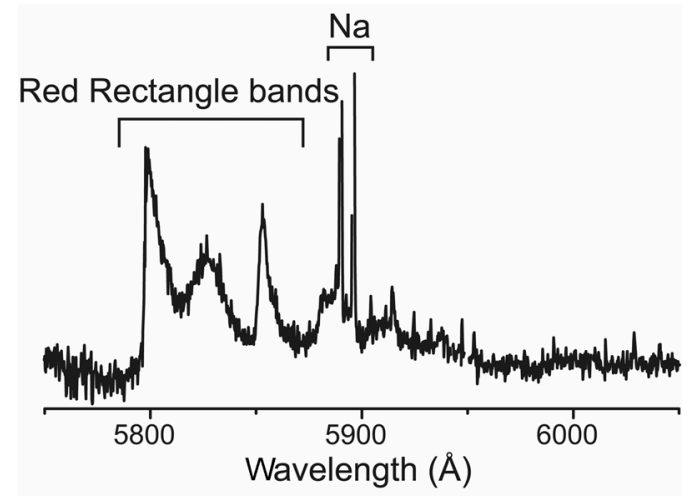

Figure 13.6 The emission spectrum from the biconial Red Rectangle nebula (below). The Red Rectangle bands are presumed to be due to as yet unidentified molecules. Spectrum courtesy of Rob Sharp, Anglo-Australian Observatory. Photograph from Hubble Heritage archive.

appears to be a red rectangle, but Hubble Space Telescope images reveal to be a beautiful biconical nebula, a view almost exactly side-on, so as to appear like a bow-tie [101]. In 1980, Gary Schmidt et al. reported molecular emission in the nebula, the most prominent series of emission being on the red side of $5800 \AA$ [102]. In 1995, Sarre noted a tantalizing coincidence between the blue edge of these emissions and several DIBs, raising the possibility that the carriers may be the same species and opening a new front in the experimental assault to uncover the carriers [103]. A crucial part of the hypothesis is that the Red Rectangle bands, being degraded to the red, are due to hotter versions of the same species as the DIB absorber. We showed, using a combination of DFT and molecular mechanics, that the broadening observed in the RRBs is consistent with large organic molecules heated at moderate temperatures of a few 10s of Kelvin, getting colder toward the edges of the nebula [104]. A suggestion by Glinski that the carrier could be phosphorescence from $\mathrm{C}_{3}$ is unlikely, yet the precise position of the lowest triplet state of $C_{3}$ remains unknown [105]. It also remains to be proven whether the RRBs actually do converge upon the DIB positions, but nevertheless, the carriers of the RRBs remain undiscovered as of 2010 and still pose a very interesting problem for 
astronomical spectroscopy [106]. As with the carriers of the DIBs, computation can aid in reducing the size of the field of candidates for experimental investigation.

\section{6}

\section{The Aromatic Infrared Bands}

As mentioned above, a set of infrared emission features observed since the 1970s [108] were suggested to be due to polycyclic aromatic hydrocarbons [89]. These bands were referred to, for a long time, as the "unidentified infrared bands," though more commonly in 2010 it is accepted to call them the "aromatic infrared bands" (AIBs). The strongest AIBs lie at 3.3, 6.2, 7.7, 8.6, 11.3, and $12.4 \mu \mathrm{m}$, which had been noted by Duley and Williams to coincide with typical vibrational frequencies of organic molecules [109]. There has been much effort dedicated to understanding the AIBs and what these emission features tell us about interstellar and circumstellar chemistry. Much of the computational work has been carried out by Charles W. Bauschlicher, Jr., at NASA, with over 40 papers on the topic. In 2008, the NASA group reported DFT calculations on PAHs as large as $\mathrm{C}_{130} \mathrm{H}_{26}$ [110]. The largest PAH ever studied spectroscopically as an isolated gas phase molecule is hexaperi-hexabenzocoronene, $\mathrm{C}_{42} \mathrm{H}_{18}$, also reported in 2008 [107]. Bauschlicher and coworkers, content to use the 4-31G basis set with the B3LYP functional, have provided the "insight" that Coulson would have requested. They suggest that the AIBs are due to a mixture of large and small PAHs, which are symmetrical and compact. Substitutions with nitrogen may improve the agreement between astronomical and synthetic spectra further still. These conclusions were supported by a 2009 report by the same authors, where irregularly shaped PAHs were studied at sizes up to $\mathrm{C}_{120} \mathrm{H}_{36}$ [111]. The spectroscopy of nitrogen containing PAHs (PANHs) is largely unexplored, especially for larger members. If these are indeed the carriers of the AIBs, it is also possible that they enter the diffuse interstellar regions and present themselves as the carriers of the DIBs. If this turns out to be the case, then it is clear that computational spectrometry would have played a significant role in guiding experimentalists toward these species.

\section{7}

\section{The Holy Grail}

One chapter is insufficient to mention every interaction between computational spectrometry and astronomical spectroscopy. However, what I have attempted to communicate above is the complexity and bilaterality of the relationship that these two fundamental fields of endeavor enjoy. It was astronomers and their laboratorybound companions who first studied the spectra of atoms and small molecules, as observed in astronomical sources such as the sun, other stars, nebulae, and comets. It was in part due to these data that led Bohr and others to propose quantized energylevel structures for atoms, but Mulliken more than others advanced our understand- 
ing of molecular structure by seeking to explain the band spectra presented by astronomers and molecular spectroscopists. His Nobel Prize in Chemistry, awarded "for his fundamental work concerning chemical bonds and the electronic structure of molecules by the molecular orbital method," in 1966, was overdue. By then computational spectrometry was beginning to take off as a subject with the possibilities afforded by the developments in computing. In 2010, computational results are used regularly for large molecules as a qualitative guide for experimental investigation, and there are some results, such as the spectroscopy of the $c^{3} \Sigma_{u}^{+}$ state of $\mathrm{C}_{2}$, where computation was the first to arrive at the correct answer. ${ }^{21)}$ Our work on $\mathrm{C}_{2}$ showed the possibility to calculate vibrational frequencies of second-row diatomic molecules to within $0.1 \%$. However, the absolute positions of electronic transitions were generally calculated to within about $0.5 \%$ of the true values. Coulson may have wanted insight rather than numbers, but in the coming years it need not be "either-or." What I will put forward is the "Holy Grail" of computational spectrometry as it relates to astronomical spectroscopy, to calculate the electronic spectra of a large organic molecule to spectroscopic accuracy. This may at first sound like an unreasonable request - because it is! Let us lay down this challenge in 2010 to calculate electronic spectra of molecules with accuracies of $1 \mathrm{~cm}^{-1}$ or better on each band. We have shown that this is still not possible for $\mathrm{C}_{2}$ in a single calculation, but a series of calculations that isolate the effects of basis set size and level of correlation may be found to achieve the task. Just as the list of known molecules in space had zero members in 1940, so is our list of accurately calculated electronic spectra in 2010, some 70 years later. The author sincerely hopes that in the next 70 years of his life (by which time he will be 105!) he will see computational spectrometry develop to the stage where calculations of electronic spectra make redundant the careful collection of laboratory spectra. The only pity would be that playing with lasers, vacuums, and discharges is a lot of fun. Let us begin.

\section{References}

1 Young, T. (1802) Philos. Trans. R. Soc. Lond., 92, 12.

2 Young, T. (1801) Philos. Trans. R. Soc. Lond., 91, 23.

3 Herschel, W. (1802) Philos. Trans. R. Soc. Lond., 92, 213.

4 Wollaston, W.H. (1802) Philos. Trans. R. Soc. Lond., 92, 365.

5 Kirchhoff, G. and Bunsen, R. (1860) Annalen der Physik und der Chemie, 110, 161.

6 Swan, W. (1857) Trans. R. Soc. Edinb Earth, 21, 411.
7 Huggins, W. (1864) Philos. Trans. R. Soc. Lond., 154, 139.

8 Donati, G.B. (1864) Astron. Nachr., 62, 375.

9 Huggins, W. (1868) Philos. Trans. R. Soc. Lond., 158, 529.

10 Huggins, W. (1874) Proc. R. Soc. Lond., 23, 154.

11 Huggins, W. (1881) Proc. R. Soc. Lond., 33, 1.

12 Liveing, G.D. and Dewar, J. (1880) Proc. R. Soc. Lond., 30, 494.

13 Ångström, K. and Thalén, T.-R. (1875) Nova Acta Reg. Soc. Sc. Upsal., 3, 9.

21) Both the calculations and the experiments were undertaken in my group. Although the calculations were essentially finished before the experiments had begun, they were in press at a later date. 
14 Fowler, A. (1910) Mon. Not. R. Astron. Soc., 70, 176.

15 Fowler, A. (1910) Mon. Not. R. Astron. Soc., 70, 484.

16 Birge, R.T. (1926) Phys. Rev., 28, 1157.

17 van Vleck, J.H. (1927) J. Opt. Soc. Am., 15, 201.

18 MacKellar, A. (1940) Proc. Astron. Soc. Pacific, 57, 187.

19 Słyk, K., Bondar, A.V., Galazutdinov, G.A., and Krełowski, J. (2008) Mon. Not. R. Astron. Soc., 390, 1733.

20 Roth, K.C., Meyer, D.M., and Hawkins, I. (1993) Astrophys. J., 413, L67.

21 Jackson, W.M. (1974) J. Chem. Phys., 61, 4177.

22 Cartwright, D.C. and Hay, P.J. (1982) Astrophys. J., 257, 383.

23 Davis, S.P., Shortenhaus, D., Stark, G., Engelman, R., Jr., Phillips, J.G., and Hubbard, R.P. (1986) Astrophys. J., 303, 892.

24 Bauschlicher, C.W., Jr., Langhoff, S.R., and Taylor, P.R. (1988) Astrophys. J., 332, 531.

$25 \mathrm{Lu}, \mathrm{R} ., \mathrm{Huang}, \mathrm{Y}$, and Halpern, J.A. (1992) Astrophys. J., 395, 710

26 Johnson, R.C. (1927) Philos. Trans. R. Soc. Lond. A, 226, 157.

27 Mulliken, R.S. (1927) Phys. Rev., 29, 637.

28 Johnson, R.C. and Asundi, R.K. (1929) Proc. R. Soc. Lond. A, 124, 668.

29 Mulliken, R.S. (1939) Phys. Rev., 56, 778

30 Ballik, E.A. and Ramsay, D.A. (1959) J. Chem. Phys., 31, 1128.

31 Clementi, E. (1960) Astrophys. J., 132, 898 .

32 Mulliken, R.S. (1953) J. Phys. Chem., 56, 295.

33 Clementi, E. (1960) Astrophys. J., 133, 303.

34 Souza, S.P. and Lutz, B.L. (1977) Astrophys. J., 216, L49.

35 Roux, F., Cerny, D., and d'Incan, J. (1976) Astrophys. J., 204, 940.

36 Chaffee, F.H., Jr. and Lutz, B.L. (1978) Astrophys. J., 221, L91.

37 Arnold, J.O. and Langhoff, S.R. (1978) J. Quant. Spectrosc. Radiat. Transf., 19, 461.

38 Zeitz, M., Peyerimhoff, S.D., and Buenker, R.J. (1978) Chem. Phys. Lett., 58, 487.
39 Chabalowski, C.F., Buenker, R.J., and Peyerimhoff, S.D. (1981) Chem. Phys. Lett., 83, 441.

40 Chabalowski, C.F., Peyerimhoff, S.D., and Buenker, R.J. (1983) Chem. Phys., 81, 57.

41 van Dishoeck, E.F. and Black, J.H. (1982) Astrophys. J., 258, 533.

42 Kokkin, D.L., Bacskay, G.B., and Schmidt, T.W. (2007) J. Chem. Phys., 126, 084302.

43 Bragg, A.E., Wester, R., Davis, A.V., Kammrath, A., and Neumark, D.M. (2003) Chem. Phys. Lett., 376, 767.

44 Davis, S.P., Abrams, M.C., Phillips, J.G., and Rao, M.L.P. (1988) J. Opt. Soc. Am. B, 5, 2280.

45 Kokkin, D.L., Reilly, N.J., Morris, C.W., Nakajima, M., Nauta, K., Kable, S.H., and Schmidt, T.W. (2006) J. Chem. Phys., 125, 231101

46 Joester, J.A., Nakajima, M., Reilly, N.J., Kokkin, D.L., Nauta, K., Kable, S.H., and Schmidt, T.W. (2007) J. Chem. Phys., 127, 214303.

47 Glancy, A.E. (1919) Astrophys. J., 49, 196.

48 Raffety, C.W. (1916) Philos. Mag. Ser., 6 (32), 546.

49 Bobrovnikoff, N.T. (1931) Astrophys. J., 73, 61.

50 Swings, P., Elvey, C.T., and Babcock, H.W. (1940) Astrophys. J., 94, 320.

51 Herzberg, G. (1942) Astrophys. J., 96, 314.

52 Monfils, A. and Rosen, B. (1949) Nature, 164, 713.

53 Douglas, A.E. (1951) Astrophys. J., 114, 446.

54 Clusius, K. and Douglas, A.E. (1954) Can. J. Phys., 32, 319.

55 Gausset, L., Herzberg, G., Lagerqvist, A., and Rosen, P. (1965) Astrophys. J., 142, 45.

56 Liskow, D.H., Bender, C.F., and Schaefer, H.F., III (1972) J. Chem. Phys., 56, 5075.

57 Saha, S. and Western, C.M. (2006) J. Chem. Phys., 125, 224307.

58 Perić-Radić, J., Römelt, J., Peyerimhoff, S.D., and Buenker, R.J. (1977) Chem. Phys. Lett., 50, 344.

59 Chabalowski, C.F., Buenker, R.J., and Peyerimhoff, S.D. (1986) J. Chem. Phys., 84, 268. 
60 Maier, J.P., Lakin, N.M., Walker, G.H., and Bohlender, D.A. (2001) Astrophys. J., 553, 267.

61 Becker, K.H., Tatarczyk, T.A., and Perić-Radić, J. (1979) Chem. Phys. Lett., 60, 502.

62 Zhang, G., Chen, K.-S., Merer, A.J., Hsu, Y.-C., Chen, W.-J., Shaji, S., and Liao, Y.-A. (2005) J. Chem. Phys., 122, 244308.

63 Monninger, G., Förderer, M., Gürtler, P., Kalhofer, S., Petersen, S., Nemes, L., Szalay, P.G., and Krätschmer, W. (2002) J. Phys. Chem. A, 106, 5779.

64 Oka, T., Thorburn, J.A., McCall, B.J., Friedman, S.D., Hobbs, L.M., Sonnentrucker, P., Welty, D.E., and York, D.G. (2003) Astrophys. J., 582, 823.

65 Cernicharo, J., Barlow, M.J., and Gonzalez-Alfonso, E. et al. (1996) Astron. Astrophys., 315, L201.

66 Cernicharo, J., Goicoechea, J.R., and Caux, E. (2001) Astrophys. J., 534, L199.

67 Cleeton, C.E. and Williams, N.H. (1934) Phys. Rev., 45, 234.

68 Herschberger, W.D. (1945) J. Appl. Phys., 17, 495.

69 Becker, G.E. and Autler, S.H. (1946) Phys. Rev., 70, 300.

70 Cheung, A.C., Rank, D.M., Townes, C.H., Thornton, D.D., and Welch, W.J. (1969) Nature, 221, 626.

71 Cheung, A.C., Rank, D.M., Townes, C.H., Thornton, D.D., and Welch, W.J. (1968) Phys. Rev. Lett, 21, 1701.

72 McCarthy, M.C. and Thaddeus, P. (2001) Chem. Soc. Rev., 30, 177.

73 Thaddeus, P. and McCarthy, M.C. (2001) Spectrochim. Acta A, 57, 757.

74 Kawaguchi, K., Kasai, Y., Ishikawa, S., and Kaifu, N. (1995) Publ. Astron. Soc. Jpn., 47, 853.

75 Aoki, K. (2000) Chem. Phys. Lett., 323, 55.

76 McCarthy, M.C., Gottlieb, C.A., Gupta, H., and Thaddeus, P. (2006) Astrophys. J., 652, L141.

77 Cernicharo, J., Guélin, M., Agúndez, M., Kawaguchi, K., McCarthy, M., and Thaddeus, P. (2007) Astron. Astrophys., 467, L37.

78 Gupta, H., Brünken, S., Tamassia, F., Gottlieb, C.A., McCarthy, M.C., and
Thaddeus, P. (2007) Astrophys. J., 655,

L57.

79 Brünken, S., Gupta, H., Gottlieb, C.A., McCarthy, M.C., and Thaddeus, P. (2007) Astrophys. J., 664, L43.

80 Heger, M.L. (1922) Lick Observatory Bull., 10, 146.

81 Merrill, P.W. (1936) Astrophys. J., 83, 126

82 Smith, W.H., Snow, T.P., Jr., and York, D.G. (1977) Astrophys. J., 218, 124.

83 Douglas, A.E. (1977) Nature, 269, 130.

84 Frenking G., Kim, K.S., and Scuseria G.E. (eds) (2005) Theory and Applications of Computational Chemistry: The First Forty Years, Elsevier.

85 Ding, H., Schmidt, T.W., Pino, T., Boguslavskiy, A.E., Güthe, F., and Maier, J.P. (2003) J. Chem. Phys., 119, 814.

86 Ball, C., McCarthy, M.C., and Thaddeus, P. (2000) Astrophys. J., 525, L61.

87 Mühlhäuser, M., Haubrich, J., and Peyerimhoff, S.D. (2002) Chem. Phys., 280, 205.

88 Coulson, C.A. and Rushbrooke, G.S. (1940) Proc. Camb. Philol. Soc., 36, 193.

89 Léger, A. and Puget, J.L. (1984) Astron. Astrophys., 137, L5.

90 Léger, A. and d'Hendecourt, L. (1985) Astron. Astrophys., 146, 81.

91 van der Zwet, G.P. and Allamandola, L.J. (1985) Astron. Astrophys., 146, 76.

92 Crawford, M.K., Tielens, A.G.G.M., and Allamandola, L.J. (1985) Astrophys. J., 293, L45.

93 Weisman, J.L., Lee, T.J., and HeadGordon, M. (2001) Spectrochim. Acta A, 57, 931.

94 Hammonds, M., Pathak, A., and Sarre, P.J. (2009) Phys. Chem. Chem. Phys., 11, 4458.

95 Stecher, T.P. (1965) Astrophys. J., 142, 1683.

96 Braga, M., Larsson, S., Rosen, A., and Volosov, A. (1991) Astron. Astrophys., 245, 232.

97 Chhowalla, M., Wang, H., Sano, N., Teo, K.B.B., Lee, S.B., and Amaratunga, G.A.J. (2003) Phys. Rev. Lett., 90, 155504.

98 Malloci, G., Mulas, G., Cecchi-Pestellini, C., and Joblin, C. (2008) Astron. Astrophys., 489, 1183. 
99 Cecchi-Pestellini, C., Malloci, G., Mulas, G., Cecchi-Pestellini, C., Joblin, C., and Williams, D.A. (2008) Astron. Astrophys., 486, L25.

100 Cohen, M., Anderson, C.M., Cowley, A., Coyne, G.V., Fawley, W.M., Gull, T.R., Harlan, E.A., Herbig, G.H., Holden, F., Hudson, H.S., Jakoubek, R.O., Johnson, H.M., Merrill, K.M., Schiffer, F.H., Soifer, B.T., and Zuckerman, B. (1975) Astrophys. J., 196, 179.

101 Cohen, M., van Winckel, H., Bond, H.E., and Gull, T.R. (2004) Astron. J., 127, 2362.

102 Schmidt, G.D., Cohen, M., and Margon, B. (1980) Astrophys. J., 239, L133.

103 Sarre, P.J., Miles, J.R., and Scarrott, S.M. (1995) Science, 269, 674.

104 Sharp, R.G., Reilly, N.J., Kable, S.H., and Schmidt, T.W. (2006) Astrophys. J., 639, 194.
105 Glinski, R.J. and Nuth, J.A. (1997) Astrophys. Space Sci., 249, 143.

106 Glinski, R.J., Michaels, P.D., Anderson, C.M., Schmidt, T.W., Sharp, R.G., Sitko, M.L., Bernstein, L.S., and van Winckel, H. (2009) Astrophys. Space Sci., 323, 337.

107 Kokkin, D.L., Troy, T.P., Nakajima, M., Nauta, K., Varberg, T.D., Metha, G.F., Lucas, N.T., and Schmidt, T.W. (2008) Astrophys. J. Lett., 681, L49.

108 Gillett, F.C., Forrest, W.J., and Merrill, K.M. (1973) Astrophys. J., 183, 87.

109 Duley, W.W. and Williams, D.A. (1981) Mon. Not. R. Astron. Soc., 196, 269.

110 Bauschlicher, C.W., Jr., Peeters, E., and Allamandola, L.J. (2008) Astrophys. J., 678, 316.

111 Bauschlicher, C.W., Jr., Peeters, E., and Allamandola, L.J. (2009) Astrophys. J., 697, 311. 